\title{
EL CULTO A NABIA EN HISPANIA Y LAS DIOSAS POLIFUNCIONALES INDOEUROPEAS
}

\author{
JUAN CARLOS OLIVARES PEDREÑO \\ Universidad de Alicante
}

\begin{abstract}
Las inscripciones relativas a Nabia en Hispania muestran algunas particularidades que la diferencian del resto de divinidades femeninas. Es la única diosa constatada en toda la amplia región situada al norte del río Duero y se alude a ella con epítetos que indican una tutela sobre núcleos poblados. Además, sus altares votivos han sido descubiertos en diversos contextos: elevaciones montañosas, fuentes o en el interior de castros. Finalmente, la diosa es citada dos veces, una de ellas sin epítetos y la otra con un apelativo, en una misma dedicación. Todos estos datos apuntan que Nabia tenía un carácter polifuncional, como algunas de las principales diosas del ámbito indo-iranio, romano o céltico insular.
\end{abstract}

Inscriptions related to Nabia in Hispania show some peculiarities which set her apart from other feminine deities. She is as the only goddess recognised throughout the whole region up to the north of the Duero river, and is referred to by terms which indicate her protection on the populated areas. Furthermore, her votive altars have been discovered in various contexts: montains, springs and inside forts. Finally, the name of the goddess is cited twice in the same dedication. All this data shows that Nabia had a multi-functional character, like some of the other main goddesses in the Indo-Iranian, Roman or Celtic insular world.

Muy pocas aportaciones teóricas se han hecho sobre la caracterización religiosa de la diosa Nabia desde que Melena le dedicó un magnífico trabajo a raíz del hallazgo del ara de la Dehesa de "El Gaitán" (Cáceres), en el que el autor intentaba perfilar la naturaleza de la deidad teniendo en cuenta su etimología y tres documentos principales: la existencia de una media luna esculpida en el ara de $\mathrm{S}$. Mamed de Lousada, la inscripción de Marecos $y$, finalmente, la inscripción y relieves de la "Fonte do Ídolo" (Braga). A partir de estas informaciones, Melena llegó a la conclusión de que Nabia sería una diosa "de los valles selvosos, de los bosques y de los montes, como la Diana latina, valles que en su hondón pudieron acoger la presencia de un río, que explicaría el hidrónimo y la caracterización de la que Nabia habría sido objeto y, muy especialmente, su vinculación con deidades acuáticas" (MELENA, 1984, 242-245).

La teoría de Melena mostraba una gran coherencia en función de la información de la que se sirvió y, por tanto, sus conciusiones son plausibles. Sin embargo, creemos disponer de otros datos no tenidos en cuenta por este autor que permitirían otorgar a esta divinidad lusitano-galaica un papel más importante en el panteón religioso y unas funciones de mayor am- plitud. El primer dato al que nos referimos tiene que ver con la distribución geográfica de los testimonios de Nabia en relación con el del resto de divinidades femeninas.

\section{EL TERRITORIO DE CULTO DE NABIA}

Melena dividía los testimonios situados al norte del Duero en tres grupos: el lucense, el orensano y el bracaraugustano al que se añadía, al sur del citado río, el grupo cacereño. Según él, Nabia habría sido una diosa bracaraugustana cuyo culto se habría difundido siguiendo las vías de comunicación hacia el norte por Aquae Flauiae y Forum Limicorum hasta Lucus Augusti y hacia Asturica Augusta y, por otra parte, por el sur hacia Augusta Emerita (MELENA, 1984, 245-248). La hipótesis que plantearemos más adelante sobre la significación de Nabia tendrá como premisa que ésta era la diosa lusitano-galaica a la que más ampliamente se rendía culto al norte del Duero y que, por tanto, su adoración en determinados ámbitos de Gallaecia no era producto de una difusión desde el área de Bracara Augusta. Con el fin de consolidar este primer argumento, hemos de hacer un breve análisis a la tesis difusionista de Melena. 
Los únicos argumentos de este investigador para presumir una dispersión del culto se fundamentan, en primer lugar, en la posibilidad de que Nabia fuera una diosa bracaraugustana en origen $y$, en segundo lugar, en la relativa cercanía de los testimonios epigráficos de la divinidad a distintas vías de comunicación. Si el primer supuesto fuera correcto, es evidente que habría que aceptar una difusión del culto habida cuenta de la dispersión de los testimonios epigráficos, pero ni el propio autor afirma con convicción el origen territorial de la diosa: "si Nabia es una diosa bracaraugustana, se ha difundido su culto siguiendo unas vías bien definidas" (MELENA, 1984, 248). En cuanto al segundo argumento, tampoco implica una difusión ni ofrece pistas sobre su posible origen por lo que, en definitiva, no existe ningún apoyo sólido que permita considerar la tesis como probable (GARCíA FERNÁNDEZ-ALBALAT, 1988, 256-257). En cambio, creemos que existe suficiente base para afirmar que, al menos en los territorios al norte del Duero, el culto a esta diosa estaba ya implantado mucho tiempo antes de que los altares votivos que hoy conocemos fueran realizados.

En primer lugar, de las restantes divinidades masculinas o femeninas cuyos testimonios aparecen en el área de Bracara Augusta, no conocemos ninguna cuyo culto sea constatable en otras regiones donde sí aparecen altares dedicados a Nabia. Por tanto, en el improbable supuesto de que los testimonios de esta diosa en las regiones lucense y orensana fueran producto de una difusión del culto a partir de desplazamientos de población desde el área bracaraugustana, el de Nabia sería el único caso conocido según los datos de que disponemos. De hecho, el culto a Cosus, que es el más representado en la zona de Braga entre las divinidades masculinas, ofrece una dispersión geográfica contrapuesta a la de Nabia, por toda la región costera desde el Cabo de Finisterre hasta Viseu y, desapareciendo en toda la Galicia central y en los distritos de VilaReal y Bragança, se constata de nuevo en la comarca leonesa del Bierzo. Así pues, si hubo un movimiento de individuos que llevó consigo el culto a Nabia, es paradójico que estos grupos sólo difundieran la adoración a esa diosa y no a las divinidades restantes de su panteón hacia la Gallaecia central y el área extremeña.

En segundo lugar, el altar ofrecido a Nabia Sesmaca en Puebla de Trives, Orense
(CIL II 2602; BLÁZQUEZ, 1962, 180; TRANOY, $1981,294)$ vincula a la diosa, por medio de otra inscripción hallada en la misma localidad, con un castellum Sesm [...]' del que sería divinidad tutelar. Por consiguiente, no es muy probable que una diosa procedente de otro lugar haya acabado convirtiéndose en la divinidad protectora de un núcleo de población por encima de las autóctonas (GARCÍA FERNÁNDEZ-ALBALAT, 1988, 257).

Más aceptable parece la tesis de que los testimonios cacereños de Nabia sean producto de un desplazamiento del culto desde el área bracarense, ya que no existen testimonios en un área intermedia donde han aparecido numerosos hallazgos epigráficos de divinidades autóctonas, como es el distrito de Castelo Branco y el sur de Guarda, en los que conocemos muchos nombres de divinidades femeninas indígenas como Trebaruna, Arentia, Munis, Erbina y Oipaengia. En este sentido, parece lógico pensar que si el culto a Nabia hubiera estado sólidamente implantado en todo este ámbito, habría dejado algunos testimonios. Sin embargo, tampoco los argumentos que otorgan una procedencia norteña al culto cacereño de Nabia son determinantes.

En resumen, la tesis de una difusión del culto de Nabia en múltiples direcciones desde el foco bracarense adolece de falta de argumentos demostrativos $y$, en realidad, aporta algunos problemas insolubles. Los datos disponibles llevan, desde nuestro punto de vista, más en la dirección de considerar que todo el territorio donde se ubican los epígrafes de esta divinidad al norte del Duero sería un área original de culto, en el caso de que sea lícito utilizar este término.

Hemos de tener en cuenta la evidencia de que, en todo el ámbito lusitano-galaico al norte del citado río, la diosa que tratamos es la más representada por testimonios epigráficos con mucha diferencia sobre las restantes, por lo que aún parece más improbable que Nabia, si hubiera sido una divinidad procedente del área bracarense, se hubiera impuesto sobre la totalidad de las deidades femeninas al norte del Duero. Este último argumento nos devuelve a nuestro principal objetivo: observar la relación de Nabia con el resto de divinidades in-

${ }^{1}$ CIL II 2601; MELENA, 1984, 237 n²: Nauiae / Ancetolus / Auri(ensis) exs c(astellum) / Sesm(acorum?) / uotum []/ possit [ ]/q(oius) e(um) c(ompotem) f(ecit) []. 
dígenas femeninas con el fin de acercarnos a su caracterización religiosá.

\section{NABIA Y LAS DIVINIDADES FEMENINAS DE GALLAECIA}

Vaya por delante una primera y muy importante evidencia que, paradójicamente, no ha sido apuntada hasta el momento: en todo el territorio noroccidental de la Península Ibérica, Nabia es la única diosa indígena supra-local conocida ${ }^{2}$. El resto son de alcance local y cada una de ellas se ha constatado por un sólo testimonio que, en varios casos, son de dudosa comprensión.

En la región de Braga conocemos tres inscripciones seguras alusivas a Nabia. En la primera de ellas, descubierta en Marecos (Penafiel, Porto) la diosa aparece citada dos veces, como Nabia Corona y, posteriormente, con la denominación Nabia sin apelativo (LE ROUX y TRANOY, 1974, 252-255). Otras dos dedicaciones a Nabia en esta región proceden de "Monte Baltar" (Vandoma, Paredes) entre Porto y Penafiel (CIL II 2378; VASCONCELOS, 1905,277 ) y de Braga (TEIXEIRA, 1938, 151153; CORTEZ, 1952, 32-45).

Además de estos testimonios cuya lectura del teónimo es correcta, tenemos otras dos inscripciones sobre las que existen algunos problemas de lectura o interpretación. La primera de ellas, procedente de Orjais (Braga) se dedicó, según algunos autores a Ameipicri sacrum (VASCONCELOS, 1905, 333; BLÁZQUEZ, 1962, 169; ENCARNAÇÃO, 1975, 87-88) y, según otros, a Ambieicri sacrum (TRANOY, 1980, 75). Posteriormente, Melena interpretó las primeras letras de ese supuesto teónimo como A(ugustae) Nabie..., suponiendo un nexo na en lugar de $m$ (MELENA, 1984, 256, $\mathrm{n}^{\circ} 18$ ). Respecto a la segunda inscripción, hallada en Braga, se planteó un problema parecido: el teónimo era leído como Ambiorebi (LE ROUX y TRANOY, 1973, 201-202, $\left.\mathrm{n}^{\circ} 12\right)$, pero Melena lo definió como A(ugustae) Nabi(e)... (MELENA, 1984, $\left.256 n^{\circ} 7\right)$. No obstante, la interpretación de los teónimos permanece dudosa, puesto que no existen argumentos que impidan su consideración como apelativos.

En este ámbito aparecen otros epígrafes donde el teónimo se puede considerar como dudoso: el primer caso es el de Abne, diosa a la que pudo aludirse como $D$ (eae) $D$ (ominae) A(ugustae) (VASCONCELOS, 1913, 214-215; CARDOZO, 1935, 24, nº 19; BLÁZQUEZ, 1962,
219; ENCARNAÇÃO, 1975, 77-78) O D(eae) D(ominae) Nostrac) (TRANOY, 1981, 268; GARCÍA, 1991, $n^{\circ} 1$ ). Por otra parte, Encarnação creía que la lectura correcta podía ser Abnai y Abascal veía la posibilidad de que se tratara de otra mención a Nabia ${ }^{3}$.

El segundo posible teónimo es Frouida, leído Por Pereira-Caldas en una "columnilla moldurada" que se halló junto a la parroquia de S. João do Souto (Braga) y actualmente desaparecida; dado que sólo él la leyó antes de su desaparición existen dudas sobre este testimonio (PEREIRA-CALDAS, 1885 = OLIVEIRA, 1985, 33-34) que, para Hübner, se podía tratar de una dedicación a Fortuna, con el apelativo [P]rouida (HÜBNER, EE 8, 116). También se plantean muchas reservas sobre la mención a una diosa Lida en el altar de Marecos ${ }^{4}$.

Además de las dedicaciones a Nabia, es segura la mención a la divinidad femenina Ocaera, que se halló en la iglesia de S. João do Campo, en las Terras do Bouro, dominadas por la Sierra de Gerês (CIL II 2458; CRUZ, 1972, 105-108). También es muy probable la denominación Calaicia, leída en una pieza de Sobreira, Paredes, en el distrito de Porto (TRANOY, 1977, 225 ss.). Por tanto, sólo tenemos en el noroeste de Portugal dos menciones seguras a divinidades femeninas que, por otra parte, aparecen una sola vez y son unimembres, por lo que podrían ser apelativos citados con omisión del teónimo. En este sentido, nombres como Calaicia que aluden a demarcaciones territoriales o étnicas suelen funcionar más como epítetos que como teónimos en la epigrafía votiva de Europa occidental.

En la provincia de Orense la preeminencia del culto a Nabia es, si cabe, más patente. Los testimonios de esta divinidad son cuatro, procedentes de S. Juan de Camba (Castro Caldelas) en el que se otorga a la divinidad el

\footnotetext{
2 Hasta hace unos años se pensaba que existía una segunda diosa adorada en varios lugares de Gallaecia: Couentina, con dos inscripciones conocidas. Sin embargo, recientemente se ha impuesto la tesis de considerar estas dedicaciones como pertenecientes al dios Cohue o Cuhue (PRÓSPER, 1997,288 ss.).

${ }^{3}$ ENCARNAÇÃO $\left(1970,209-211, n^{\circ} 1\right)$. Según ABASCAL $(1995,86)$, la lectura de los apelativos y el teónimo podía ser d(eae) d(ominae) N/abiae.

${ }^{4}$ Algunos autores que estudiaron la pieza leían ese teónimo (LE ROUX y TRANOY, 1974, 252-255 y MELENA, 1984, 238) pero, posteriormente, se planteaban dudas a la primera parte del teónimo, que LE ROUX (1994, 560 ss.), interpretando el teónimo como [I]dae, relacionaba con Cibeles.
} 
apelativo Elaesurraeca (BLÁZQUEZ, 1962 , 179; RODRÍGUEZ COLMENERO, 1987, 151$151, n^{\circ} 87$ ), Nocelo da Pena (FITA, 1911, 402404; LORENZO y BOUZA, 1965, 150-151, $\mathrm{n}^{\circ}$ 80) y los dos, ya citados, de Puebla de Trives en uno de los cuales se alude a Nabia como Sesmaca.

Junto a estos altares orensanos dedicados a Nabia, sólo podemos citar una inscripción se alude, probablemente, a otra divinidad femenina: Proinetia, en Folgoso, Xinzo de Limia (RODRÍGUEZ COLMENERO, 1987, n 92). No obstante, también es algo dudoso el carácter teonímico de la mención, puesto que conocemos un epígrafe votivo dedicado a un Lar cuyo segundo apelativo guarda muchas semejanzas con la denominación citada, Circeiebaecus Proenetiaegus (RIVAS, 1973, 83-87; RODRÍGUEZ COLMENERO, 1987, 210-211 $\mathrm{n}^{\circ}$ 122). Es posible que Proinetia fuera un apelativo utilizado para aludir a la divinidad que, por tanto, permanecería desconocida.

Finalmente, en la provincia de Lugo conocemos tres altares dedicados a Nabia que fueron descubiertos en S. Martín de Montemeda (BOUZA Y D'ORS, 1959, 27, $n^{\circ} 7$; ARIAS et al., 1979, 90-92, $n^{\circ}$ 71), S. Mamed de Lousada (BOUZA Y D'ORS, 1959, $26 \mathrm{n}^{\circ}$ 6; ARIAS et al., $\left.1979,92-93, n^{\circ} 72\right)$, ambos en el concejo de Guntín y, finalmente, en S. Miguel de Orbazai (ARIAS, 1984, 229-233). En esta provincia sólo conocemos otro teónimo femenino seguro, Rea, conocido por un testimonio de la capital lucense (ARIAS et al., 1979, 34-35, $n^{\circ} 7$ ). El otro posible teónimo aparecido en la capital lucense es Poemana (ARIAS et al., 1979, 33-34, $n^{\circ}$ 6), aunque lo podemos considerar extremadamente dudoso a causa del desgaste de la inscripción.

El último testimonio de Nabia al norte del Duero (RODRÍGUEZ COLMENERO, 1987, 200, $\left.n^{\circ} 114\right)$, apareció en el distrito portugués de Vila-Real (Tres Minas, Vila Pouça de Aguiar), en cuyo territorio sólo consta otra posible dedicación a una divinidad femenina, aunque las dificultades de lectura impiden asegurar si se trata de una diosa indígena (RODRÍGUEZ COLMENERO, 1987, 147-148, $\mathrm{n}^{\circ} 85$ ).

El balance que surge de los datos epigráficos referentes a divinidades femeninas indígenas del área lusitano-galaica al norte del río Duero es muy sorprendente, por cuanto aparece una sola divinidad muy representada, Nabia, de la que contabilizamos once inscripciones sin tener en cuenta otras dudosas que algunos autores han adjudicado a esta deidad ${ }^{5}$.
Por otra parte, conocemos ocho dedicaciones más hechas a diosas de las que cuatro son de lectura o interpretación dudosas. Las de lectura segura son Ocaera, Rea, Calaicia y Proinetia pero, sin embargo, son unimembres. Por ello, es posible que algunas de estas menciones, sobre todo las dos últimas, fueran epítetos. En cualquier caso, aún considerando válidas todas las denominaciones dudosas, persiste la evidencia de que sólo Nabia goza de una difusión supra-local.

Ante estos datos, hemos de cuestionarnos si existe una sustancial falta de información epigráfica en este ámbito que pudiera justificar la constatación de una sola diosa adorada en un amplio territorio.

En un sondeo cuantitativo de las inscripciones aparecidas en la zona que probablemente mencionan a divinidades indígenas, observamos que se acercan a las doscientas. De éstas, se constatan cinco teónimos masculinos supra-locales, algunos de ellos muy representados: Cosus (ocho inscripciones), Bandua (ocho), Reue (diez), Lug (tres) y Aernus (tres); por otra parte, conocemos denominaciones locales que se pueden caracterizar como teónimos con seguridad por aparecer en alguna ocasión acompañados de apelativos: Vestius (dos inscripciones), Lariberus (doce), Verore (cuatro) y algunos más representados por una inscripción. Considerando estos datos en conjunto observamos que, aunque son de menor cuantía que en otras zonas (OLIVARES, 1999a, 286 ss.), son bastante relevantes para considerar que la presencia de Nabia como única divinidad femenina supra-local representada al norte del Duero no viene dada, al menos exclusivamente, por una escasez de información.

Tampoco debemos pensar que en el territorio que estudiamos otras diosas indígenas se ocultan bajo nombres de divinidades romanas puesto que, aunque se dan en esta región más casos de interpretatio epigráfica que en otros ámbitos de la Península Ibérica, sobre todo referidos a Lares y Genios con apelativos indígenas (OLIVARES, en prensa), los referentes a deidades femeninaș son muy escasos. De

\footnotetext{
${ }^{5}$ Nos referimos a los ejemplos citados de Ambiorebi y Ambieicri que MELENA $\left(1984,256 n^{\circ} 7\right.$ y 18) adjudicó a Nabia y, por otra parte, a una inscripción de S. Román de Cervantes (Lugo) de la que sólo se leen las dos últimas letras del teónimo. ARIAS et al. $(1979,14)$. lo restituyen así: [Naui]ae /[sacru]m.
} 
hecho, en la región donde se constata el culto a Nabia al norte del Duero sólo son conocidas cuatro inscripciones más que aluden a diosas romanas individuales con epítetos indígenas: Juno Veamuaearum, Juno Meirurnarum y dos que se dedican a Tutela con epítetos alusivos a localidades. Ahora bien, sólo las dos dedicaciones a Tutela son fiables (EE 8, 111 y MENOR, 1979, 305-306), ya que las menciones a Juno (CIL II 2409a y CIL II 430) son de lectura dudosa y las piezas están hoy desaparecidas.

Aunque siempre hemos de considerar posible una aparición de nuevos datos que provocara un vuelco sustancial a nuestros enfoques creemos probable, por el momento, que el panorama que nos ofrece la información disponible muestra los perfiles de la estructura panteística indígena de la zona. Ahora bien, si aceptáramos este esquema nos surge de inmediato un problema de gran importancia, ya que en todo el territorio ocupado por los distritos portugueses de Castelo Branco y Guarda así como en la región española de Extremadura al norte del Tajo existen varias divinidades femeninas que compartían el culto mostrando una estructura que contrasta de manera clara con la existente al norte del río Duero.

En ese ámbito, que guarda una sustancial coherencia interna desde el punto de vista teonímico, se constatan varios teónimos femeninos seguros de carácter regional, ya que frecuentemente van acompañados de apelativo $\mathrm{y}$, además, aparecen en el mismo ámbito territorial: Arentia (cuatro inscripciones), Trebaruna (nueve) y Munis (tres). Además, aparecen dos testimonios algo distanciados entre sí de otras divinidades, Laneana y Erbina, además de algunos teónimos locales. Por otra parte, en la región extremeña al sur del Tajo aparece un esquema similar, pero formado por distintos teónimos, con treinta y seis dedicaciones a Ataecina, cuatro a Lacipaea y otras cuatro a Nabia (OLIVARES, 1999c, 116-117).

Por tanto, si rechazamos la falta de datos para explicar la sola existencia de Nabia como diosa supra-local al norte del Duero queda una única alternativa: los grupos sociales que habitaban Gallaecia habrían adjudicado a Nabia un carácter polifuncional otorgándole un rol religioso de amplio espectro que, en otros ámbitos, ejercían varias divinidades con nombres diferentes. Ahora bien, para comprobar esta hipótesis deberíamos confirmar que existen en los testimonios referentes a Nabia en Gallaecia aspectos característicos que se refieran a diversos tipos funcionales.
NABIA COMO DIVINIDAD POLIFUNCIONAL

Una de ios primeros aspecios de Nábia que hemos de resaltar es la diversidad de contextos geográficos y arqueológicos en que aparecen los testimonios epigráficos. Algunos de ellos se hallaron en parajes de monte, en ocasiones en elevaciones montañosas separadas de núcleos poblados.

Un ejemplo claro de este tipo de ofrendas es el procedente de Marecos (Penafiel, Porto), en el que se citan sacrificios hechos a la diosa indígena Nabia, en una ocasión sin epíteto y en otra con el apelativo Corona y, por otra parte, a Júpiter (LE ROUX y TRANOY, 1973, 252-255). Pinho describía el momento del descubrimiento del ara de Marecos del siguiente modo: "Ao passar um dia em Marecos, junto da capelinha da Senhora do Destêrro, divisou no meio dum monte de pedras e entulho um plinto de granito com una inscrição que não pôede ler" (PINHO, 1928, 96-97). Este autor dejaba patente el lugar despoblado del hallazgo conjeturando, por tanto, que la capilla de la Virgen se habría construido sobre las ruinas de un santuario de Nabia. Es muy probable que bajo el teónimo Júpiter se haga referencia a un dios indígena o, cuando menos, que Júpiter había asimilado o sustituido en ese lugar sagrado a otra divinidad autóctona de características similares, puesto que se asocia a la diosa lusitano-galaica. Por otra parte, la ofrenda se lleva a cabo en un paraje montañoso alejado de núcleos poblados.

La inscripción alusiva a Corono procedente de Cerzedelo (Guimarães, Braga) ofrece algunas informaciones de interés que nos pueden ayudar a comprender mejor la dedicación de Marecos y, por otra parte, a vislumbrar con más nitidez el significado religioso de Nabia. La pieza fue hallada en el lugar de Castro, en un campo de la propiedad Ilamada Vila Mea. Según Sarmento, "o lugar ocupa a coroa do Monte de Pedrados, que pelo norte e sofrivelmente abrupto e pelo nascente liga com o sistema orográfico, onde domina o alto cabeço da Senhora do Monte" (SARMENTO, 1933, 301-303). Por tanto, se trataría de una pieza vinculada a una emblemática elevación montañosa. Por otra parte, junto a la iglesia de Cerzedelo, muy cerca de Castro, se halló un ara dedicada a Júpiter Optimo Máximo que, 
además, tenía la particularidad de un nexo $M A$ idéntico al del altar ofrecido a Corono (SARMENTO, 1933, 302).

Si es lícito inferir, desde un punto de vista metodológico, que unas aras dedicadas a dioses romanos e indígenas halladas en un mismo lugar nos indiquen una relación o identificación entre esas divinidades en el caso citado se dan, además, algunas coincidencias que no parecen casuales: Corona aparece como apelativo de una diosa que se vincula a Júpiter en la inscripción de Marecos, mientras que el testimonio de Corono aparece a $30 \mathrm{Km}$. de este enclave en una montaña y muy cerca de un altar dedicado al dios supremo de los romanos con el que guarda similitudes formales.

Las mismas conclusiones parecen apuntarse en otra inscripción de Figueira de Castelo Rodrigo (Guarda). Se trata de un altar dedicado a Coruae que, fuera esta denominación un similar teónimo o un epíteto, debe guardar una cierta relación con los citados anteriormente, ya que contienen el mismo radical (CURADO, $1985,650-651$ ). Es digno de resaltar que la pieza procede de un contexto paisajístico comparable al de los anteriores epígrafes, ya que se halló en la cumbre de la Sierra de Marofa cuando se construyó en el lugar la capilla de Nuestra Señora de Fátima. Además, cabe resaltar que en el centro del coronamiento de este altar ofrecido a Coruae se representó un creciente lunar, al igual que en un ara lucense de Nabia (TRANOY, 1981, 293).

Otros testimonios de la diosa Nabia aparecieron en lugares de similares características. La dedicación efectuada por Cicerón, hijo de Manco, en Pedrogão Pequeno (Sertá, Castelo Branco) se halló en la cima de una colina en la que existía una iglesia "feita com pedras da torre que os Romanos tinhão tido não longe da dita igreja". Según las Memorias Parroquiales de 1758, "toda a planicie do simo deste monte se acha cereada de hum muro de pedra todo ja arruinado e pela sua antiguidade mostra ser feito antes que os Mouros entrarem nas Hespanhas para propugnaculo ou defença das gentes que ahy estavão aquarteladas, pois não mostra que dentro delle houvessem cazas, porem ainda se conhece, onde estava a porta do dito muro" 6 .

La inscripción hallada en la Dehesa de El Gaitán (Cáceres) estaba en plena Sierra de San Pedro y había aparecido entre los restos arruinados de "El Castillo", donde se habian efectuado hallazgos superficiales de monedas y fragmentos de cerámica (MELENA, 1984,
233). El epígrafe de Vandoma (Paredes, Porto) fue encontrado en "Monte Baltar" (HÜBNER, $1871,78)$ y conocemos otro altar ofrecido a Nauia procedente del atrio de la iglesia de $\mathrm{S}$. Pedro, en el Monte do Viso, Nocelo da Pena (LORENZO Y BOUZA, 1965, 150) 7 , donde había sido hallada junto a otra inscripción ilegible. Es posible que las tres aras halladas en la finca "La Cardosa del Mayoralgo", en la Sierra de S. Pedro y muy cerca del Puerto de la Mezquita (Cáceres) también se hubieran dedicado a Nabia (MELENA, 1984, 258-259).

Otros testimonios de la diosa se encontraron en un contexto muy diferente, en clara relación con comunidades pobladas de distinta entidad. El altar de S. Martín de Montemeda procedía del castro del Picato de donde había sido recogida "junto a otras piedras sueltas" (BOUZA y D'ORS, 1959, 27). También el ara de $S$. Mamed de Lousada se enmarcaba en las cercanías de ese mismo castro. Por ello, Arias, Le Roux y Tranoy afirmaban que el culto a Nabia Arconunieca estaría relacionado con un grupo concreto de población sobre el que ejercería de divinidad tutelar (ARIAS et al., 1979, 92-93). Por otra parte, la inscripción de S. Miguel de Orbazai se encontró en "un camiño ó pé da muralla exterior do castro de Penarrubia" (ARIAS, 1984, 229). En el mismo contexto se sitúan las dedicaciones de Trujillo, Cáceres ${ }^{8}$ y también el ara de Braga (TEIXEIRA, 1938, 151 ss.). Pero ésta última no se vinculaba sólamente a la ciudad, sino que se halló junto a la Fonte do ídolo relacionándose también con las aguas.

En resumen, observamos como los testimonios referentes a Nabia se hallaron en contextos de diferente carácter; en primer lugar, en probables santuarios ubicados en parajes despoblados donde ocupaban colinas o mon- 
tañas boscosas; en segundo lugar, en castros o ciudades $y$, por último, junto a una fuente sagrada.

Los datos ofrecidos por los textos epigráficos apuntan la misma tendencia que muestran los lugares de hallazgo. El altar dedicado a Nabia Sesmaca evidenciaba el carácter de la diosa como tutelar del castellum Sesm(acorum?) y, posiblemente, Nabia Arconunieca sería la diosa protectora de la comunidad residente en el Castro de "O Picato". Pero para valorar estas dedicaciones en su justa medida hemos de tener en cuenta algunos factores: la única divinidad femenina romana que aparece en toda la región occidental al norte del Duero con epítetos referentes a núcleos de población indígenas es Tutela, con los epítetos Tiriensis, Berisis que se refiere probablemente al castellum Beriso que se conoce por la inscripción de S. Juan de Godán (Salas, Oviedo) y Bolgensis ${ }^{9}$. Además, cuando estos epítetos aluden a divinidades masculinas hispanas, se refieren a los Genios tutelares y, en las provincias galas, casi en todas las ocasiones aluden a Marte (OLIVARES, 1999b, 281 ss.). Estos datos nos permiten inferir que uno de los aspectos de Nabia en Gallaecia era el de diosa tutelar de grupos de población y que, como ocurría con este tipo de divinidades, su protección tendría también una vinculación con la guerra.

Por otra parte, hemos de tomar en consideración que la diosa es citada en primer lugar y por dos veces junto a Júpiter en la inscripción de Marecos. Este hecho implicaría que Nabia tendría "un rôle cosmique qui englobe non seulement les eaux mais encore le ciel et la terre" (LE ROUX y TRANOY, 1974, 255). Según algunos autores, Nabia Corona podría indicar una paredría con Corono, dios conocido por otra inscripción. Sin embargo, no es seguro que Corono sea un teónimo y es evidente que Corona es un epíteto. Por tanto, la vinculación más directa posible se observa en el propio altar sacrificial de Marecos y se establece entre Nabia y Júpiter. Esta simple yuxtaposición de sus dedicaciones permite inferir una paredría, puesto que a la diosa se efectúan las dos primeras ofrendas, a Júpiter la tercera y, si la lectura ...urgo que aparece después de Júpiter corresponde a la parte final de otro teónimo, este sería masculino.

En definitiva, en el ara de Marecos Nabia habría ejercido el papel de diosa relacionada con el dios supremo Júpiter. En este punto se hace necesario citar la inscripción de Trujillo que, aunque muy dudosa, relacionaría a Nabia con Salamati, dios asociado al monte Jálama (1492 m.) y, además, citado con el apelativo Optimo $^{10}$.

Estos datos, aunque sin confirmación expresa, apuntan un aspecto de Nabia diferente al que habíamos observado en las inscripciones lucenses donde salía a la luz su carácter tutelar y se vinculaba a poblaciones concretas por medio de apelativos o por el lugar de hallazgo de los testimonios. Su relación con Júpiter en el ara de Marecos y, en menor medida, el hecho de que algunos de sus lugares de culto estuvieran en montañas o colinas alejadas de núcleos poblados la vinculan, por otra parte, con la soberanía.

La doble ofrenda a la diosa en el altar sacrificial de Marecos adquiere una mayor significación si consideramos que Nabia, al norte del Duero, era considerada una divinidad polifuncional. Otra posible explicación podría considerar a las dos Nabiae como una misma diosa adorada en dos enclaves distintos, pero esta tesis tiene dos objeciones: la primera es que una de las dos ofrendas se hace a Nabia sin apelativos, por lo que no se evidencia una advocación epicórica concreta y, en segundo lugar, no aparecen dedicantes individuales o comunitarios de diferentes ámbitos.

Por tanto, la doble mención a Nabia en la misma pieza votiva indica más bien que bajo ese nombre se adoraban dos entidades divinas diferentes desde el punto de vista funcional: una Nabia soberana relacionada con Júpiter y otra versión de la divinidad más directamente vinculada a la tutela y defensa de una comunidad o a aspectos más privados como la salud, la fertilidad o la riqueza.

Es probable que esta idea se plasmara en las abreviaturas que dan comienzo a la inscripción, que Le Roux y Tranoy interpretaban O(ptimae) V(irgini) Co(nseruatrici uel rniferae) et Nim(phae) Danigom. Estos autores hacían al respecto en 1974 las siguientes considera-

9 Sobre las dos primeras, aparecidas en Torre de Pinhão (Sabrosa) y S. Vicente de Coucieiro (Paderne, Orense) uid. supra. El castellum Beriso se cita en C/L II 5739. En cuanto a la última, fue hallada en Cacabelos del Bierzo, León (BLÁZQUEZ, 1962, 63; GÓMEZ MORENO, 1979, 59; DIEGO SANTOS, 1986, $\mathrm{n}^{\circ} 62$ ).

10 MELENA, 1985, 475 ss.; en la pieza de Trujillo, RODRÍGUEZ (1878, 146-147), que es el único autor que la vio, leía lo siguiente: Salam / ac / Nabi / llucius / u s / a; Melena la interpretó así: Salam(ati)/ac/Nabi(e)/llucius / u s.la. 
ciones: "Au début, les abréviations sont inhabituelles mais devaient correspondre à un formulaire connu qui seul peut expliquer le choix d'abréger les mots. Le sens et la structure du texte, la nécessité de rapporter l'expression Nim Danigom pour Danigo(ru)m à Nabia Corona impliquent que les termes $\mathrm{O}$. V. CO soient des qualificatifs de cette même divinité" (LE ROUX y TRANOY, 1974, 253).

Desde este punto de vista, Tranoy profundizaba en un estudio posterior en el sentido teológico de las abreviaturas: "La première fois, Nabia est entourée d'un ensemble de qualificatifs qui en fait une déese assimilée aux Nymphes et protectrice d'une communauté, les Danigi. Ce rapprochement entre Nabia et les Nymphes exprime une des fonctions essentielles de Nabia, deése de la nature, liée aux eaux qui abondent dans cette région. Elle apparaît alors comme une divinité chargée de veiller sur le peuple des Danigi et de lui assurer à la fois de bonnes récoltes et de beaux troupeaux" (TRANOY, 1981, 281-282). Con una interpretación de la segunda dedicación a la diosa, Tranoy culminaba una hipótesis muy valiosa sobre el carácter religioso de Nabia: "dans ce cas, ce n'est plus la déesse considérée dans son action purement locale auprès des Danigi que les fidèles veulent honorer, mais la grande divinité Nabia, au rôle cosmique débordant le cadre régional et citée pour cette raison juste avant Jupiter" (LE ROUX y TRANOY, 1974, 281282).

El investigador francés diferenciaba entre dos diosas Nabia desde el punto de vista funcional pero, principalmente, desde el punto de vista de la amplitud territorial de su culto: "L'autel de Marecos se situe donc sur deux plans: le premier est le reflet du maintien des traditions locales autour d'un groupe, les Danigi, avec la notion de hiérarchie des divinités, fondée sur le principe de dieux tutélaires locaux et de dieux à rayonnement plus vaste, ces deux élements pouvant se confondre pour une même divinité comme Nabia Corona et Nabia" (LE ROUX y TRANOY, 1974, 281-282).

Como muestran los textos mitológicos y religiosos de otros pueblos indoeuropeos, el criterio fundamental que caracterizaba a una divinidad y la insertaba en un esquema jerarquizado era su función. La plasmación local o regional de su culto era tan sólo una consecuencia de la misma. De este modo, la Nabia asociada a Júpiter sería una diosa soberana perteneciente, al igual que la Nabia tutelar de la localidad, al esquema panteístico de la comu- nidad de los Danigi. En el resto de las comunidades de Gallaecia podía reproducirse este esquema, ello sin perjuicio de que cuando se quería evocar el carácter protector y tutelar de una diosa se hiciera en referencia a la comunidad local y cuando se hacía referencia a su función soberana no fuera necesario efectuar tal reducción.

En esencia, Nabia era una sola divinidad $y$ el hecho de que sea la única femenina que aparece en un amplio territorio al norte del río Duero vendría dada por su carácter polifuncional, que respondería al amplio abanico de necesidades religiosas de la población galaica. Con todo, las características de Nabia, tal como nos son vislumbradas por los datos, no se diferencian de las que se conocen de otras divinidades femeninas de origen indoeuropeo.

\section{DIOSAS POLIFUNCIONALES EN LAS MITOLOGÍAS INDOEUROPEAS.}

Muchas celebraciones romanas que tenían por objeto la sexualidad y la fecundidad de las mujeres eran presididas por Juno, como las Nonae Caprotinae que acontecían el 7 de Julio en las que se aludía a la diosa con el apelativo Caprotina o las Matronalia, que se celebraban en las Calendas de Marzo, en las que el elemento principal era la participación femenina. Todas las Calendas estaban consagradas a Juno, que presidía el inicio de los meses con el "renacimiento" de la luna.

No obstante, además de los ritos relacionados con la fertilidad, a Juno se dedicaban otras conmemoraciones en las que se resaltaba su carácter político-religioso. La Juno Curitis de Tibur era una diosa que cumplía un papel protector y guerrero, con atributos como el escudo y el carro, al igual que la Juno de Lanuvio que iba provista de lanza y escudo. En palabras de Dumézil, "Marchante, agressive, le torse bombé, ou bien montée sur un char au galop, la lance en avant, tendant du bras gauche un bouclier à double échancrure analogue à celui des Salii, le type de déesse que montrent statues et monnaies est clairement et uniquement guerrier" (DUMÉZIL, 1987, 304).

El carácter trifuncional de Juno se hace patente en algunas inscripciones en las que se hace un voto a Iunoni Seispiti Matri Reginae. Para Dumézil, el apelativo Regina mostraría el papel político-religioso de la diosa; Mater estaría relacionado con la función plasmada en las Matronalia, relacionada con la fecundidad fe- 
menina; Seispes aludiría al carácter guerrero de la divinidad. En definitiva, Juno sería una divinidad capaz de asumir simultáneamente las tres funciones de la ideología religiosa indoeuropea: la realeza sagrada, la fuerza guerrera y la fecundidad, $\sin$ perjuicio de que en sus diferentes advocaciones pudiera ser resaltado alguno de estos aspectos sobre los demás (DUMÉZIL, 1987, 305).

En la religión indo-irania, la védica Sarasvati es una diosa-río que presenta también varios aspectos. Asociándose a las divinidades masculinas características del tercer nivel funcional, los gemelos Asvin, en numerosos himnos o ritos es proveedora de toda clase de riquezas ( $R V 2,41,17$ y 10, 131, 5; DUMÉZIL, 1977, 95; BHATTACHARYYA, 1983, 21), de las aguas que fertilizan los campos cultivables, de la salud, la descendencia y la vitalidad (RV 1, 164, 49; DUMÉZIL, 1987, 308; BHATTACHARYYA, 1983, 21-22). En este sentido, es calificada como la "primera de las madres, el mejor de los ríos y la más grande de las diosas" ( $R V 2,41,16)$. Pero también es guerrera, destructora de enemigos, por lo que se le atribuye un apelativo propio del dios guerrero Indra: "destructora de resistencias, victoriosa" (RV 6, 61, 7; DUMÉZIL, 1987, 308; BHATTACHARYYA, 1983, 23). Por otra parte, asume las características más típicamente sacerdotales del primer nivel: es pura y purificadora y "reina de todos los pensamientos piadosos" ( $R V 1$ 1, 3, 10-11; DUMÉZIL, 1987. 308).

Progresivamente, Sarasvati se identificó con Vac, la personificación de la sabiduría y la elocuencia, que se asociaba a todos los dioses del panteón védico: "yo estoy asociada con los Rudras, los Vasus, los Adityas y los Visvadevas. Yo sostengo tanto el sol como el océano (Mitra y Varuna), el firmamento (Indra), el fuego (Agni) y a los dos Asvins... Yo soy la reina, la que confiere los tesoros, la detentora del conocimiento..." (RV, 10, 125, 1-8; BHATTACHARYYA, $1983,25)$. Esta asociación a varios dioses, sus múltiples funciones así como el origen celestial de las dos diosas, relacionadas con el rayo y el trueno, generaron su final identificación como una sola diosa, ya en la literatura védica tardía (BHATTACHARYYA, 1983, 27).

De modo similar explicaba Dumézil uno de los principales acontecimientos narrados en el Mahabharata, el matrimonio poliándrico de Draupadi con los cinco hijos de Pandu. Estos son presentados como reflejos de los grandes dioses masculinos funcionales del panteón védico y, cada uno de ellos, muestra el mismo carácter que su modeio en correspondencia al lugar que ocupa en el esquema funcional de la religión indo-irania. Por tanto, el hecho de que Draupadi contraiga matrimonio con los cinco Pandava sería una transposición a la epopeya del modelo estructural del panteón, en el que "a la lista de los dioses funcionales masculinos, en la que cada personaje o grupo de personajes representa una función y sólo una, de forma que su reunión jerarquizada presenta un análisis de la estructura de conjunto, las teorías de varios pueblos indoeuropeos yuxtaponen y asocian una diosa única pero trivalente que hace en cierta manera la síntesis de las mismas funciones" (DUMÉZIL, 1977, 98).

En el Avesta aparece otra diosa del mismo carácter, Anahita, río mítico y deidad fluvial con un carácter trifuncional claro, purificadora de la que dependía el éxito de los guerreros, la sabiduría y la santidad de los sacerdotes o el feliz alumbramiento de las mujeres. Su denominación "la húmeda, la fuerte, la inmaculada" denotaría su triple naturaleza, en sintonía con la mención a Juno Seispes Mater Regina (DUMÉZIL, 1977, 96).

La consideración de la divinidad femenina como una y, simultáneamente, triple es un fenómeno recurrente y de una gran importancia en el ciclo mitológigo irlandés (MAC CANA, 1983, 42-43). En estos textos se citan tres divinidades llamadas Macha que aparecen con caracteres diferenciados: la primera es una vidente que predice el futuro; la segunda es una diosa guerrera y la tercera está estrechamente ligada a la fecundidad ${ }^{11}$. De Vries opinaba, en sintonía con las tesis de Dumézil, que estas tres diosas representaban las tres funciones sociales de los celtas (DE VRIES, 1963, 137).

También las diosas Morrigán y Badbh, que forman en ocasiones trío con Macha, son percibidas como una sola deidad o como tres (GREEN, 1992, 138 y 154-155). En el Táin Bó Cuailnge, el nombre de Badbh aparece sólo como una réerencia específica a la metamorfosis de Morrigán en pájaro, pero esta distinción no pudo mantenerse durante mucho tiempo y cuando los escribas de época medieval

\footnotetext{
"Según GREEN (1995, 40-41 y 77), "like other sovereigntyfigures, the Morrigán was a multi-functional and many layered character. War, death, prophecy, guardianship, sexuality, fertility and rulership were all inextricably intertwined in this complex divinity, whose relationship to humans appears to have been capricious and changing, but who was strongly committed to the fortunes of Ireland.
} 
plasmaron las referencias en los textos, Badbh y Morrigán habian sido ya convertidos en dos nombres intercambiables sobre los que, además, los prejuicios de los sacerdotes cristianos que compilaron los textos en el siglo XI habían resaltado sus aspectos más sombríos y demoniacos (HERBERT, 1996, 145-148).

La naturaleza de Morrigán se muestra en la Batalla de Mag Tuired, que narra la confrontación entre dos grupos de divinidades por el control de la tierra irlandesa, donde se cita un encuentro sexual de Morrigán ("Gran Reina") con el dios máximo de ese pueblo, el Dagda. Este texto presenta a la diosa ante la aparición del dios con las piernas abiertas situadas a ambos lados de un río, una de ellas al sur del mismo y la otra al norte, indicando simbólicamente su dominio sobre la totalidad de la tierra (HERBERT, 1996, 142-143). Después de su cópula, Morrigán ofrece al Dagda su ayuda en la batalla.

Por tanto, dejando a un lado matices siniestros reflejados por los copistas medievales, se pueden observar diversos perfiles de la divinidad: su carácter soberano derivado de su nombre y de su vinculación al rey de los dioses (GREEN, 1995, 76 ss.); en segundo lugar su función tutelar y guerrera y, finalmente, su directa relación con la tierra de Irlanda y la fecundidad, representada por su poliandria y promiscuidad sexual (ROSS, 1986, 128; GREEN $1995,43-45)^{12}$. En definitiva, se trataría de una deidad de múltiples aspectos y funciones.

Otra deidad semejante, si no equivalente, era Eriu, epónima de la tierra irlandesa e hija de Dealbaeth, quien en otro lugar aparece como el padre de Dana, reforzando el paralelo entre las dos diosas (DE VRIES, 1963, 136). Los textos no citan tres diosas de nombre Eriu, sino que aluden a ella junto a otras dos diosas: Banba y Fotla; estas tres divinidades tenían por esposos a Mac Gréine, Mac Cuill y Mac Cecht estructurando un modelo comparable al del ejemplo ya citado de las tres Macha. En cualquier caso, estaríamos ante otra diosa unitaria y, a su vez, triple que resumía toda la variedad de aspectos que los pueblos celtas encomendaban a sus divinidades femeninas.

En este sentido, el número tres no era sólo la expresión de la plenitud del poder, sino que reflejaba una repartición de la esencia total de la divinidad entre sus fundamentos principales. Estos mitos, paralelos al que mostraba el citado matrimonio poliándrico de Draupadi resume, en palabras de De Vries, "lidée que les dieux des diverses fonctions (royauté, armée et agriculture) ont dû s'unir à la Mère Irlande sous trois formes leur correspondant respectivement, a fin que chacun pût vivre sur cette île selon sa nature propre, de façon profitable" (DE VRIES, 1963, 165). En un último análisis, el carácter de esta divinidad mostraba su papel protector contra todas las formas del mal que pudieran afectar a la tierra patria y a sus pobladores: contra la enfermedad, la invasión o el hambre (GREEN, 1995, 70 ss.).

A la misma categoría de estas diosas y, probablemente equivalente a ellas, pertenecía otra deidad Británica: Brigit ${ }^{13}$. Era una divinidad y, a la vez triple, ya que existían tres hermanas con el mismo nombre de las que, cada una de ellas, mostraría uno de los aspectos de la actividad o función total (VENDRYES, 1949, 277). Brigit se muestra como una diosa de los poetas, de los comerciantes y de la prosperidad, con un fuerte perfil estacional y purificador. En este sentido, se le dedicaba el día 1 de Febrero la celebración de $/ \mathrm{mbolc}$, muy relacionada con la fertilidad.

Esta diosa aparece testimoniada en siete inscripciones de época romana como Brigantia (GREEN, 1992, 50; ROSS, 1986, 125). En una inscripción aparecida en Castleford (CIL VII 200; RIB 607) y en otra hallada en Greetland (RIB 628), ambas de Yorkshire, la diosa es aludida como Victoria Brigantia; en otra hallada en Castlesteads (Cumbria) se le cita como Dea Nympha Brig(antia) en un voto pro salute et incolumitate del emperador efectuado por un procurator Augusti (CIL VII 875; RIB 2066; BIRLEY, 1986, 66); en otro epigrafe de Corbridge (Northumberland) la ofrenda es dedicada a Júpiter Aeternus Dolichenus et Caelestis Brigantia (RIB 1131). Estas inscripciones muestran, por tanto, tres aspectos claramente diferenciados de la diosa: una función relacionada con la guerra que se plasma mediante la yuxtaposición de su nombre al de Vic-

\footnotetext{
12 Para HERBERT $(1996,145)$, el carácter guerrero, tradicionalmente adjudicado a esta diosa, no sería un aspecto primario; más relevantes serían su función soberana y su relación con la fertilidad de la tierra y el ganado.

${ }^{13}$ Anu, Danu y Brigit se confundirían entre sí y serían tres nombres de una misma concepción divina (SJOESTEDT, 1949, 45; VENDRYES, 1949, 277). Para LE ROUX Y GUYONVARC'H $(1993,117)$, también serían la misma diosa, como encarnaciones de la tierra madre aunque con denominaciones diferentes Boand, epónima del río Boyne o río sagrado de la Irlanda celta, Etain, Eithne y Tailtiu. Para GRENIER $(1923,109)$, todas las diosas galas serian hipostasias diversas de la gran diosa madre primitiva, la Tierra. En el mismo sentido, ROSS $(1986,125)$.
} 
toria; otra que tiene que ver con la salud y, en tercer lugar, un carácter astral vinculado a Júpiter Dolicheno que la relaciona con Caelestis, diosa estelar de origen semítico (HENIG, 1984, 236-239).

Además, en un testimonio procedente de Birrens (Northumberland), en cuyo texto Brigantia no se asocia explícitamente a divinidades romanas, consta una representación de la diosa con las trazas de Minerva, pero alada con una cabeza de Gorgona sobre su seno y un casco con plumas rodeado de una corona turriforme; en su mano derecha sostiene una lanza, en su izquierda una bola; en este mismo lado se asienta su escudo ( $R I B$ 2091; BIRLEY, 1986, 66). Esta escultura, como ya habíamos observado a partir de las inscripciones, resume en sí misma la fusión de atributos de diferentes tipos de divinidad: la corona turriforme de Tyche, las alas de Victoria, el casco y la égida de Minerva y el betilo de Caelestis (HENIG, 1984, 239) ${ }^{14}$.

En definitiva, se confirma algo que apuntan los ciclos mitológicos y que sigue la tendencia que habíamos propuesto para Nabia: Brigantia es una diosa polifuncional, lo que explica que sea la divinidad femenina más ampliamente testimoniada desde el punto de vista territorial. Pero la importancia del culto a Brigantia adquiere unas connotaciones, en lo que respecta a su relación con otras diosas britanas, que lo asemejan en mayor medida al de Nabia.

Hemos de tener en cuenta que gran número de las diosas que se testimonian en epígrafes hallados en Gran Bretaña son originales del continente, desde donde habrían llegado a partir del principado de Claudio. En este sentido, Bémont efectuó un análisis de las divinidades procedentes de uno y otro lado del Canal de la Mancha, estableciendo cerca de una treintena de teónimos masculinos o femeninos atestiguados en ambas orillas del mismo que habrían sido llevados por los soldados a Gran Bretaña (BÉMONT, 1981, 69) ${ }^{15}$ y otras divinidades que sólo son constatables en la isla pero que, con toda probabilidad, proceden del continente ${ }^{16}$. Finalmente, otras diosas fueron introducidas por iniciativa de la administración imperial17.

En resumen, se pueden considerar diosas indígenas britanas Latis o Ratis; Sulis ${ }^{18}$, Couentina $^{19}$, Arnemetia, divinidad epónima del establecimiento termal ubicado en Aquae Arnemetiae (Buxton, Dérbyshire) ${ }^{20}$; Ancasta, Cuda (BIRLEY, 1986, 67), Saitada ${ }^{21}$ y Verbeia ${ }^{22}$.
Por tanto, además de Brigantia, la única diosa indígena de la provincia que consta en los tes-

\begin{abstract}
${ }^{14}$ No obstante, algunos de estos atributos pueden acompañar a otras divinidades, como la égida, que se asocia también a Apolo, Juno o Júpiter (DAREMBERG y SAGLIO, 1969, I-1, 101); la corona turriforme también es atributo de Cibeles y otras divinidades tutelares ciudadanas (SZILAGYI, en VV.AA, 1966, 1038; GREEN, 1995, 196).

${ }^{15}$ En cuanto a las diosas, Epona podría haber sido traída por los soldados, puesto que sólo se testimonia en tres epígrafes (BÉMONT, 1981, 73) de los más de sesenta conocidos; la diosa Nemetona que se cita junto a Mars Loucetius en Bath (Avon) es original, sin duda, del territorio de los Tréviros (en torno a Trier, Alemania) donde son frecuentes estos dos dioses formando paredría; el altar dedicado a la dea Viradecthis fue realizado por unos habitantes del pagus Condrustis (Condroz, en Bélgica) que militaban en una cohors Tungrorum (RIB2108; BÉMONT, 1981, 78); las ofrendas a las Suleuiae tendrían su origen en las provincias de Germania Superior e Inferior (BIRLEY, 1986, 53); las dedicaciones a las Matres en las islas nunca llevan un apelativo que pueda indicar un origen britano, más bien al contrario, suelen referirse a hechos religiosos externos a la isla o denotan una nacionalidad continental, como las Matres Germanae, Afrae, Italae, Gallae, Ollototae o Transmarinae (BÉMONT, 1981, 81 y 86-87)
\end{abstract}

${ }^{16}$ La ofrenda a las deae Alaisiagae la realizaron unos ciues Tuihanti (Twente, Holanda), de una unidad de auxiliares Frisones (RIB 1593 y 1594; BÉMONT, 1981, 84-85); las deae Alateruiae fueron adoradas por una cohorte de Tungros $(R / B$ 2135; BÉMONT, ibid., 85), al igual que la diosa Ricagambeda (RIB 2107; BÉMONT, ibid., 85; BIRLEY, 1986, 75); a la dea Garmangabis rindieron culto una uexillatio de Suevos (RIB 1074; BEMONT, ibid., 85); también Harimella es de origen continental (BÉMONT, ibid., 85); la dedicación a Setlocenia pudo ser hecha por un individuo procedente de Germania, aunque no es seguro (BIRLEY, 1986, 71).

${ }^{17}$ La dea Britannia, como se puede inferir a partir de los dedicantes de los dos altares conocidos, un liberto imperial en York y un prefecto de cohorte en el muro de Antonino (BIRLEY, 1986, 66-67), era la personificación de la provincia $y$, por tanto, un producto de la propaganda oficial del Estado romano. En las monedas, se representaba por primera vez en los años 119-122 d.C. sentada y apoyada en un escudo, o bien como una amazona vestida con un traje autóctono, con lanza y escudo (ROCHETTI, en VV.AA., 1959 , 178; HENIG, 1986, 167-168)

${ }^{18}$ Todas las dedicaciones a esta diosa proceden del balneario de Bath, Avori, de las que tres la relacionan con Minerva (BIRLEY, 1986, 54).

${ }^{19} \mathrm{Se}$ conocen 14 dedicaciones a esta diosa, todas ellas en Carrawburgh (Northumberland). Dos de ellas la relacionan con las ninfas (RIB 1526 y 1527; BIRLEY, 1986, 46) lo que explica su relación con el establecimiento termal.

${ }^{20}$ Esta divinidad sería la misma que aparece en Brough-onNoú, Derbyshire (CIL VII 393; RIB 841; BÉMONT, 1981, 86 n. 128) llamada Arnomecta.

${ }^{21}$ La dedicación se hizo por la curia Textouerdorum, quizá perteneciente a los Brigantes (BIRLEY, 1986, 71).

${ }^{22}$ Posiblemente relacionada con el río local Wharfe (BIRLEY, $1986,73)$. 
timonios epigráficos y excedía del ámbito local es Latis que, por otra parte, sólo se testimonia en torno al muro de Hadriano ${ }^{23}$.

Estos datos nos ofrecen un esquema muy semejante al de Gallaecia, con una sola diosa cuyo culto abarca un amplio espacio geográfico coexistiendo únicamente con diosas indígenas locales y que, por otra parte, los altares dedicados a ella indican un carácter polifuncional. En el caso de Brigantia estas conclusiones son más sólidas si tenemos en cuenta su representación iconográfica y los textos de los ciclos mitológicos referidos a Brigit, puesto que no disponemos de datos tan determinantes sobre Nabia en Hispania.

Pero no son éstos los únicos elementos comunes entre ambas divinidades: Brigit habría dado lugar a diversos hidrónimos como el Braint, en Anglesey, o el Brent, en Middlessex (RIVET et al., 1979, 277; MAIER, 1998, 47), mientras que conocemos varios ríos en la Hispania occidental de nombre Navia. Por otra parte, la asimilación de Brigantia a Caelestis establece otro importante paralelo con Nabia, a saber: Caelestis, a pesar de su asimilación con Juno, Venus, Cibeles e Isis, fue ante todo una deidad virgen ${ }^{24}$. Este hecho la relaciona con la deidad hispana si el apelativo $V(\ldots)$ de la inscripción de Marecos se interpreta como lo hicieron Le Roux y Tranoy: V(irgini).

A la vista de los datos expuestos, nos parece muy probable que el carácter absolutamente predominante del culto a Navia en la Hispania noroccidental esté en relación con la naturaleza polifuncional de las más importantes divinidades femeninas indoeuropeas y, entre éstas, de las del panteón celta tal como se muestran en los textos irlandeses y en determinados testimonios epigráficos e iconográficos. Esta hipótesis es más probable si tenemos en cuenta que esta particularidad es la más destacable, si cabe, de este tipo de diosas.

Ahora bien, no en todos los momentos o lugares la divinidad femenina podría asumir toda la amplia gama de funciones, sino que dependiendo de circunstancias concretas, se le podía atribuir un papel u otro rindiéndole culto junto a divinidades masculinas de función específica que serían diferentes en cada ocasión. Esta polifuncionalidad invalida parte de las objeciones de García Fernández-Albalat a la tesis de Melena sobre la vinculación de Nabia a Diana, centradas principalmente en el hecho de que la diosa romana no tenía el carácter político que se observa en algunos testimonios de Nabia (GARCÍA FERNÁNDEZ-ALBALAT, 1988, 259 ss.).

Según nuestra teoría, en esos altares donde se relaciona a la diosa lusitano-galaica con comunidades indígenas se habría resaltado su carácter tutelar y político, sin perjuicio de que en otras dedicaciones se destacaran los aspectos que pudieran relacionar a Nabia con Diana. Por tanto, es muy posible que, en determinada ocasión, Nabia pudiera ser interpretada como Diana según la tesis de Melena, pero también como Juno, muy probable en el ara de Marecos como ya había señalado Tranoy, Tutela, Victoria u otra divinidad del panteón romano mientras que, en otros casos, no fuera asociada a ninguna deidad en concreto, puesto que la diosa lusitano-galaica habría asumido múltiples funciones que aglutinaban características de diversas divinidades romanas.

\section{BIBLIOGRAFÍA}

ABASCAL, J.M. 1995: "Las inscripciones latinas de Santa Lucía del Trampal (Alcuéscar, Cáceres) y el culto de Ataecina en Hispania", AEA, 68, 31-105.

ALBERTOS, M.L. 1952: "Nuevas divinidades de la antigua Hispania", Zephyrus, 3, 49-63.

ARIAS, F. 1984: "Nova inscripción romana no museo de Lugo", Boletín del Museo Provincial de Lugo 2, 229 232.

ARIAS, F., LE ROUX, P. y TRANOY, A. 1979: Inscriptions romaines de la province de Lugo, París.

BHATTACHARYYA, K. 1983: Sarasvat§ . A study on her concept and iconography, Calcuta.

BÉMONT, C. 1981: "Observations sur quelques divinités gallo-romaines: les rapports entre la Bretagne et le continent", Etudes Celtiques, 18, 65-88.

BIRLEY, E. 1986: "The deities of Roman Britain", ANRW, vol. 18.1, 6-112.

BLÁZQUEZ, J.M. 1962: Religiones primitivas de Hispania 1. Fuentes literarias y epigráficas, Madrid.

BOUZA, F. y D'ORS, A. 1959: Inscripciones romanas de Galicia II. Provincia de Lugo, Santiago de Compostela.

CARDOZO, M. 1935: Catálogo do Museu de Arqueología da Sociedade Martins Sarmento I. Secçao lapidar e de escultura, Guimarães.

COLLINGWOOD, R.G. y WRIGHT, R.P. 1965: The Roman Inscriptions of Britain I. Inscriptions on Stone (R/B), Oxford.

\footnotetext{
${ }^{23}$ Hemos de considerar también dos diosas citadas por Dion Casio, Andate y Andrasta $(62,6,1-2$ y 62, 7, 3).

${ }^{24}$ GARCÍA Y BELLIDO, 1957, 14. La diosa aparece como Dea Virgo Caelestis (ILS 4438), Dea Magna Virgo Caelestis (CIL VIII 9796) y es llamada por San Agustín (C.Dei 4,10$)$ Dea Virgo y Virginale numen; Apuleyo (Met. 6,4$)$ la describe como Virgen sentada sobre un león; Tertulliano (Apol. 23) la llama Virgo Caelestis. Aunque paradójico, es relativamente frecuente en la mitología céltica la paredría y virginidad de una misma divinidad femenina (GREEN, 1995, 204).
} 
CORTEZ. F. 1954: "A Fonte do ldolo e o culto de Asklépius em Bracara", Bracara Augusta, 5, 90-103.

CRUZ, M.B. 1972: "A ara de S. João do Campo (Gerês)", Revista de Guimarães 82, 105-108.

CURADO, F.P. 1985: "Epigrafía das Beiras (notas e correcções -1)", Beira Alta 44, 641-655.

DAREMBERG, CH. y SAGLIO, E. 1969: Dictionnaire des Antiquités grecques et romaines, Graz.

DE VRIES, J. 1963: La Religion des Celtes, París.

DIEGO, F. 1986: Inscripciones Romanas de León, León.

DUMÉZIL, G. 1977: Mito y epopeya. La ideología de las tres funciones en las epopeyas de los pueblos indoeuropeos, Barcelona (1a edición: 1968).

DUMÉZIL, G. 1987: La religion romaine archaïque, París (1 $1^{\text {a }}$ edición: 1974).

ENCARNAÇÃO, J. 1970: "Lápides a divindades indígenas no museu de Guimarães", Revista de Guimarães 80 , 207-238.

ENCARNAÇÃO, J. 1975: Divindades indigenas sob o dominio romano en Portugal, Lisboa.

FITA, F. 1911: "Nuevas lápidas romanas de Noya, Cando, Cerezo y Jumilla", BRAH 59, 398-417.

GARCÍA, J.M. 1991: Religioes antigas de Portugal. Aditamentos e observaçoes as "Religioes da Lusitania" de J. Leite de Vasconcelos, Lisboa.

GARCÍA Y BELLIDO, A. 1957: El culto a Dea Caelestis en la Península lbérica, Madrid.

GARCÍA FERNÁNDEZ-ALBALAT, M.B. 1988: "La diosa Nabia: nueva interpretación", Actas del $1^{\circ}$ Congreso Peninsular de Historia Antigua (vol. II). Editor: G. Pereira Menaut, Santiago de Compostela, 249-261.

GÓMEZ MORENO, M. 1979: Catálogo monumental de la provincia de León, León.

GREEN, M.J. 1992: Dictionary of Celtic Myth and Legend, London.

GREEN, M.J. 1995: Celtic Goddesses: Warriors, Virgins and Mothers, London.

GRENIER, A. 1923: Les Gaulois, París.

HENIG, M. 1984: "Throne, altar and Sword: civilian religion and the roman army in Britain", en T.F.C. Blagg y A.C. King (eds.): Military and civilian in Roman Britain. Cultural relationships in a frontier province, Oxford, 227-248.

HENIG, M. 1986: "Britannia", en Lexicon Iconographicum Mythologiae Classicae (LIMC), III-1, 167-169.

HERBERT, M. 1996: "Transmutations of an irish goddess", en S. Bellington y M. Green (eds.), The concept of the goddess, London y New York, 141-151.

HÜBNER, E. 1871: Noticias Archeológicas de Portugal, Lisboa.

LE ROUX, F. y GUYONVARC'H, CH. 1993: A civilização celta, Sintra ( $1^{\text {a }}$ edición: 1990).

LE ROUX, P. y TRANOY, A. 1973: "Notes d'epigraphie romaine de Galice", Cuadernos de Estudios Gallegos, 28, 221-234.

LE ROUX, P. y TRANOY, A. 1974: "Contribution a l'etude des regions rurales del Nor-ouest hispanique au HautEmpire: deux inscriptions de Penafiel", II/ Congresso Nacional de Arqueología (vol. 1), Porto, 249-257.

LORENZO, J. y BOUZA, F. 1965: "Inscripciones romanas votivas de la provincia de Orense", Cuaderno de Estudios Gallegos, 20, 127-165.

MAC CANA, P. 1983: Celtic Mythology, Feltham ( $1^{a}$ edición, 1968).

MAIER, B. 1998: Dictionary of celtic religion and culture, Rochester (New York)

MELENA, J.L. 1984: "Un ara votiva romana en El Gaitán, Cáceres", Veleia, 1, 233-259.

MELENA, J.L. 1985: "Salama, Jálama y la epigrafía latina del antiguo corregimiento", Symbolae Ludovico
Mitxelena septiacuenario oblatae Parnolona. $475-530$.

MENOR, M. 1979: "Otra ara al dios Tutela", Boletin Auriense, 9, 305-310.

OLIVARES, J.C. 1999a: "Teónimos indígenas masculinos del ámbito Lusitano-Galaico: un intento de síntesis", Actas do Congresso de proto-história europeia. Revista de Guimarães, volumen especial $n^{\circ} 1$, Guimarães, 277-296.

OLIVARES, J.C. 1999b: "Dioses indígenas vinculados a núcleos de población en la Hispania romana", Espacio, Tiempo y Forma (Hist. Ant.), 12, 279-304.

OLIVARES, J.C. 1999c: "El panteón religioso indígena en el área extremeña", H.Ant., 23, 97-118.

OLIVARES, J.C. (en prensa): "Los dioses indígenas en el noroeste de Portugal".

OLIVEIRA, E.P. 1985: "Noticias arqueológicas de Braga", Conimbriga, 24, 5-83.

OLMSTED, G. 1994: The gods of the Celts and the Indoeuropeans, Budapest.

PARENTE, J. 1980: "Subsidios inéditos para a Historia de Tres Minas", Actas do seminario de arqueologia do noroeste peninsular (3), 131-139.

PEREIRA-CALDAS, J.J.S. 1885: "Lápide romana inédita", Commercio do Minho, $\mathrm{n}^{\circ} 1865$

PINHO, J. 1928: "A ara de Marecos", Penha Fidelis, 7, $1928,95-97$ y $124-127$

PRÓSPER, B.M. 1997: "La divinidad paleo-hispana Cossue/Coso y el dios itálico Consus", Aisn, 19, 267302.

RAMÓN, J. 1951: "De epigrafía cacereña", BRAH, 128, 165-195.

RIVAS, J.C. 1973: "Nuevas aras romanas orensanas y rectificaciones interpretarivas en torno a otros epígrafes galaico-romanos ya conocidos", Boletín Auriense, 3, 57-96.

RIVAS, J.C. 1983: "Sobre la identidad de la supuesta ara romana de las "Tricivitas", Boletín Auriense, 13, 7598.

RIVET, A.L.F. y SMITH, C. 1979: The place-names of Roman Britain, London.

RODRíGUEZ, J. 1878: "La Vettonia. Monumentos é inscripciones romanas", Boletín de la Sociedad Geográfica de Madrid, 5, 146-147.

RODRÍGUEZ COLMENERO, A. 1987: Aquae Flaviae I. Fontes epigraficas, Chaves.

ROSS, A. 1986: The pagan Celts, London.

SARMENTO, F. 1933: "Inscrições inéditas", Dispersos, Coimbra, $175-180$.

SJOESTEDT, M.L. 1949: Gods and Heroes of the Celts, London ( $1^{\mathrm{a}}$ edición: 1940).

TEIXEIRA, C. 1938: "Subsidios para o estudo da arqueología bracarense. A fonte do ídolo e o culto de Nabia", Prisma, 2.1, 145-153.

TRANOY, A. 1977: "A propos des Callaeci de Pline: Epigraphie et Peuplement", Bracara Augusta, 31, 225233.

TRANOY, A. 1981: La Galice romaine. Recherches sur le nord-ouest de la Péninsule lberique dans I'antiquité, París.

VASCONCELOS, J. L. 1905: Religioes da Lusitania II, Lisboa.

VASCONCELOS, J.L. 1913: Religioes da Lusitania III, Lisboa.

VENDRYES, J. 1948: La religion des Celtes, París.

VV.AA. 1959 y 1966: Enciclopedia dell'arte antica classica e orientale (vol. II y VII), Roma. 